\title{
REDISCOVERY OF THE COURTYARD, AS A MAJOR INTANGIBLE CULTURAL HERITAGE
}

\author{
Yiorgos Hadjichristou ${ }^{1}$ \\ Dept. of Architecture, University of Nicosia, 31 Michail Yiorgalla, 2409 Nicosia CYPRUS- hadjichristou.y@unic.ac.cy
}

KEY WORDS: rediscovery of tradition, courtyards, intangible cultural heritage, spatial typologies, flexible courtyard, system of courtyards

\begin{abstract}
:
The radical and huge scale changes in the island of Cyprus, especially of the last century, resulted in a significant disruption with the social and cultural heritage. The paper will focus on the Courtyards as an indispensable part of the intangible cultural heritage which carries invaluable 'wisdoms' stemming out form the rich history of the island, the climatic and topographical conditions, but decapitated due to the modernization brought by the British colonization and the well rooted enforcement of the urban regulations of the young government. More specifically it will concentrate on ways and mechanisms of not only how to revive this intangible architectural quality, but rather to evolve it in order to generate new spatial typologies responding to the social and cultural changes in relation to their new organizational and functional requirements.
\end{abstract}

\section{THE COURTYARD: CULTURE'S WISDOM FORGED THROUGH THE RICH HISTORY}

The wisdom of traditional architecture in small, less-advanced places like Cyprus may be expressed by its organisation around a courtyard (Papacharalambous, 2001; Pfeifer, 2008). This architectural development took shape down the centuries (Sinos 1986), relating to and reflecting the specific climatic and socioeconomic conditions, the activities and culture of a particular community (Lee, 1991; Khattab, 2001) and the levels of privacy and safety required, all of which were always subject to the external conditions brought about by different historical moments. (Danilo, 1997)

The various stages in the development of this 'core' element of Cypriot architecture are, of course, interrelated with the relevant technology, materiality and achievements of society. They can depict the level of development of its civilization and highlight the specificities of each historical period.

Due to its strategic location - as the confluence of the three continents of Europe, Asia and Africa - Cyprus attracted a series of invaders that conquered and occupied the island (Kyrris, 1996). In the last 2,000 years, Cyprus was under permanent occupation by successive kingdoms, empires and cultures, such as the Romans, Byzantines, the Francs, the Venetians and the Ottomans. Cyprus only gained its independence from British rule in 1960. Each conqueror left his mark on the traditional architecture of the island and consequently on the typology of the courtyard. (Schaar, 1995; Leventis, 2005)

Apparently, the British influence was the most radical and significant that formed the current built environment: it helped to modernise the planning of the island's settlements. On the other hand, these changes forced the traditional architectural element of the courtyard, and the way buildings were arranged around it, into extinction. It not only stifled any sort of development or evolution of traditional architecture but it abruptly disrupted the continuity from tradition to contemporary urbanscape. (Lee, 1991; Khattab, 2001; Li, 2010)
Unfortunately, these new regulations implemented by the British were adopted by the young Republic of Cyprus, and they are still in force. As a result, the merits of traditional wisdom were lost, and the new planning system, together with modernisation, brought about new typologies and spatial conditions largely irrelevant to the local climatic, cultural and social circumstances.

Rediscovery of the courtyards, through evolving instead of repeating

The current paper focuses on the notion of the courtyard, but without aiming simply to revive and reinterpret it: it attempts, rather, to focus on its evolution in a way that corresponds to the reestablishment of certain strong, still vital social characteristics of the island, while also responding to the new ways of living dictated by contemporary needs.

New approaches of the courtyard as the generator of innovative spatial conditions will be examined in this paper through a series of projects executed on the island of Cyprus by the author's architectural practice, ranging from an intervention on an already existing traditional house to a series of newly proposed, different typologies of housing projects, each in a different context.

Emphasis will be given on some characteristics that are considered important for the evolution of the courtyard organisations:

. The flexibility of the courtyard, and the achievement of diverse spatial conditions in order to respond to the ever changing social needs

. The issue of the blurring zones focussing on the thresholds such as the relationship between the in and out, private and public, old and new

. The materiality and technology, and the scale of their implementation

. The merits of the 'oasis' milieu of the courtyard deriving from the physical measures taken for enhancing the bioclimatic behaviour

. The scale and various possibilities of the evolution of the courtyard system, oscillating from generating interior design 
approach to an urban scale implementation in horizontal and vertical developments

\section{STARTING FROM THE ORIGIN: AN ATTEMPT TO RECUPERATE A TRADITIONAL COURTYARD HOUSE LITERALLY CUT IN THE MIDDLE}

\section{A Way to Heal the Amputation of a Traditional Spatial Organisation}

An essential extension of a 'truncated' traditional house regenerates the amputated original 'milieu'. The study refers to one half of a traditional house in Kaimakli, the result of dowry arrangements concerning the family property, which practically cut the house into two parts. The space that had suffered most, and which used to hold the spatial relationships of the house tightly together, acting in every way as the core of the original house, was the courtyard. What was left of it failed to maintain its inherent role as the main compositional architectural element of the house.

This 'trauma' in the original traditional courtyard typology of the house inspired the challenge for a radical approach: Not just to 'fix the wounds but to 'recuperate' the traditional house and 'convert' it into a contemporary living unit through a new typology of a system of courtyards.
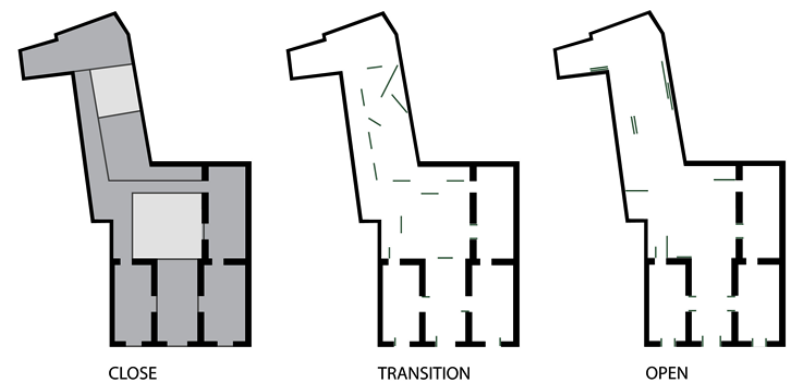

Figure 1: the three main stages of the new malleable courtyards in the traditional house

Transformation of the 'remnant' of the original courtyard into a "system of courtyards": (fig.1) This system justifies its title through the qualities given to the courtyards themselves and to their practical interaction with the surrounding spaces that define them. As a first step, the remnant of the original courtyard was transformed into two smaller courtyards that facilitate the organisation of all the spaces and the circulation of the house. This spatial composition could by itself have been an adequate intervention to this specific house. Though, a further step was taken that addressed the new courtyards in a more holistic way, in an attempt to respond to a series of new, real needs in contemporary living. The new courtyards act together as a system in conjunction with the rest of the house, which is able to transform itself into an unexpected variety of spatial conditions, ultimately unifying the whole house into one multilayered courtyard. (Tarkofsky, 1986)

Blurring zones: The original courtyard house had distinct physical borders of the indoor and outdoor spaces with the in between condition of the outdoor covered space, the so called 'sun room'. Through this adopted courtyard system, the mechanism of 'movable' surfaces that substitute the walls, doors and separation elements constantly transforms the internal and, consequently, the external spaces, mingles or merges the public and private areas, alters the movements and pauses, interchanges openness with closeness, until finally it completely 'opens up' and becomes itself the 'multi-layered' courtyard. Even at this ultimate stage, the juxtaposition of the simultaneously present dualities interweaves the resulting spatial conditions, creating an abundance of experiential environments for its users.

Furthermore, the courtyard system has tried to grapple with the levels of privacy in many ways. The continuous transformation responds to the family's ever-changing needs and therefore generates a big number of diverse spatial conditions: the entire house may be turned into an urban camping type of residence, or it can ensure the privacy of family members by creating a sequence of closed, independent spaces, or any possible combination of the two. The mixture of diverse activities simultaneously taking place, as well as the accommodation of completely disparate uses of the spaces, and their occasional transformation into a public space, in a way reflects the incessant fluidity of contemporary living in Cyprus.

Materiality - structural and natural elements as the main constituent members of a flexible, fluid courtyard: While traditional courtyards are surrounded by solid walls with openings, in this case study the materiality of the main constituent elements enhances the notion of the malleability of the courtyards. All the surfaces of the extensions are made of light materials, either glazed panes or wood panels that open or slide and, finally, almost disappear. While these architectural elements that define the courtyards disappear, the participation of the vegetation as part of this courtyard system is disclosed, gradually taking on the main role of the further, discreet levels of privacy. The vegetation is even used as material for balustrades or a swivelling shading device and becomes a vital architectural material in the system of the courtyard, while retaining its smell, texture, colours and life.

The fact that this specific case study questions and attempts to evolve the actual traditional courtyard system in its 'motherland', in other words in a listed house, generates new dynamic relationships which perhaps would not be achievable in a newly-designed contemporary building. The approach entails the merits of both the old and the current heritage, making indispensable the one to the other.
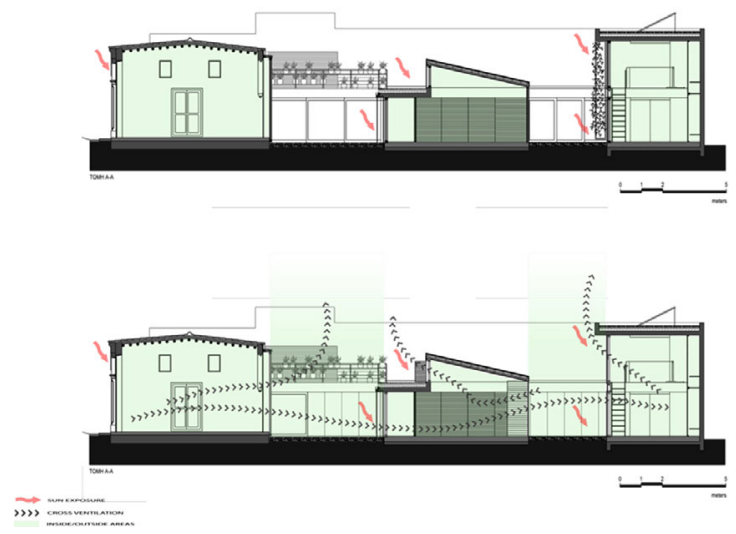

Figure 2: bioclimatic approach

Bioclimatic Approach as the Strategy of Physical Means to Generate Intangible Spatial Qualities: Originally, the decision to create a system of courtyards was in response to the climatic conditions (Serghides, D., 2010): to provide a south 
orientation for all the spaces with movable shading devices and cross ventilation. (fig.2) The amplification of the draft through the 'enforced' routes that are created in the semi and completely open stages of the house is filtered and cooled through the various shaded spaces and vegetation. The density of the light and draft is controllable by the movable elements of the courtyard, enabling the users to create the desired ambience and atmosphere of their physical oasis.

Although the qualities of contemporary life may have been to a large extent successfully transferred in this house typology, the feeling of an oasis that protects the users from the so-deemed 'negative' parameters of a modern town, like those of noise and the uncontrolled affluence of images, prevails. The user can orchestrate the desired environment by rearranging the conditions him/herself

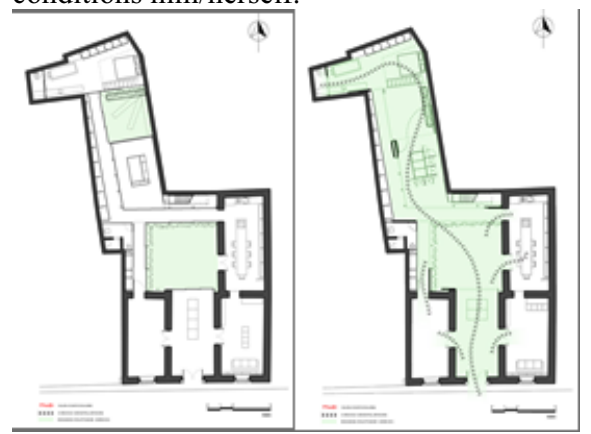

Figure 3: fluidity of spaces, activities, movements and air flow

Unexpected and Playful: The combination or overlapping of activities creates various unexpected relationships that can be enhanced by the rearrangement of the mobile constituent elements of the courtyards. The static and otherwise traditional centralised courtyard system may indeed fall into an inanimate state if it lacks the right proportions or ingredients. In this case, however, it succeeds in being transformed into a playful environment with unanticipated surprises that keep creativity alive and the mind free. These flux quality spaces (fig. 3) endlessly open up the desire for exploration and curiosity that, like a living entity, keep company to any user of the house.

Extending the courtyard system idea into the interior design: An implementation of this idea was taken a step further to the organisation of the interior spaces. A series of furniture was specifically designed for this house, which became spaces within spaces. Simulating the characteristics of the courtyards, they multiply their possibilities on different scales, incorporating further the corporeal and sensory qualities of the human being. This approach culminates in the design of the two multifunctional systems of furniture in the centre of the children's rooms. Like the courtyards themselves, they can function independently like enclosed boxes that open up to reveal various functions, such as sleeping, studying and playing, interacting first with the room and finally adding another layer to the courtyard system.

\section{IMPLEMENTATION AND EVOLUTION OF THE COURTYARD SYSTEM IN A NEW URBAN CONTEXT A CENTRAL COURTYARD AND A NETWORK OF SATELLITE PERIPHERAL COURTYARDS IN A NEW NEIGHBOURHOOD OF NICOSIA (FIG.2)}

A Central Courtyard and a Network of Satellite Peripheral Courtyards in a New Neighbourhood of Nicosia (fig.4)
A family project in a new neighbourhood of Nicosia, consisting of a main residential entity on the ground floor, two housing units for the two children of the family on the upper floor, and a working space for the teaching of Greek language and History, is organised around a central courtyard and a network of peripheral courtyards.

This newly developed part of Nicosia, like every other new development in the town, lacks any sort of identifying features. Planning regulations and the trend of building rather large homes conspire to pack houses within just three meters from the borders of the plots. The result is a sort of 'corridor' around each house, deplete of any vital outdoor spaces for family activities and suffering of any level of privacy.The character and nature of the specific users of the house and their understanding for the need of a living environment with humane qualities greatly contributed to the realisation of the proposed design, the core of which is the central courtyard. Their need for private outdoor spaces that neither faced, nor could be viewed from, neighbouring houses generated the idea of combining a central courtyard with satellite peripheral courtyards.

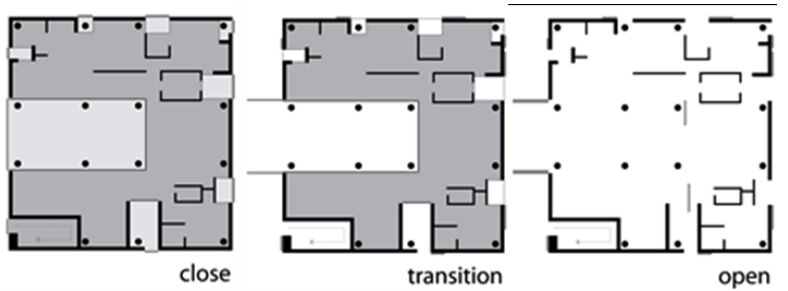

Figure 4: the 3 main phases of the courtyard system

Central courtyard: The public activities of the main residence gravitate around the central courtyard, which is defined by a transparent glazed material that enables the spaces to communicate with each other as well as with the outdoor space. For most of the year, the envelope of the courtyard vanishes through the 'shell' and out of the 'sculpted box' of the building, unifying the interior and exterior spaces. The outdoor activities mingle with the indoor ones while new dynamic relationships between them are forged.

Peripheral courtyard: The concrete shell of the 'box building' is sculpted with notches that function as a system of a peripheral network of courtyards with sliding perforated wood panels (fig.5). The panels, when closed, assure a level of privacy for all the internal spaces, since there is not a single opening on the actual skin of the building. When the panels are open, a dialogue with the surroundings opens up, reflected vividly in the transformation of the elevations. The system of courtyards acts as the facilitator between the built environment and nature and it is actually the only contact with the surrounding environment.

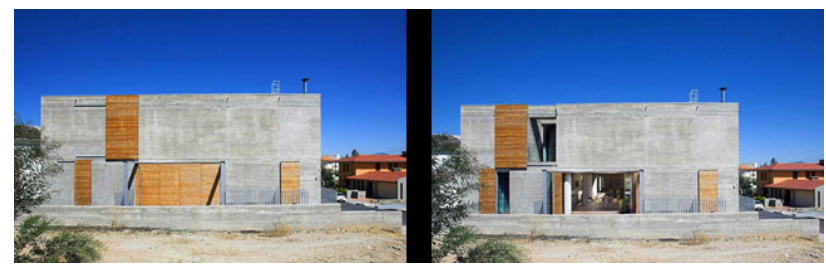

Figure 5: reflection of the peripheral courtyards on the elevations 
Collaboration between the central courtyard and the network of peripheral courtyards: The system of small peripheral courtyards works together with the central one to subtly animate the interior spaces with controlled and diffused light, helping to achieve an almost 'sacred', soothing ambience in the house. It also manages to refresh and cool the spaces with continuous cross ventilation. Through the transformable envelope of the 'sculpted box' with its sliding panels, the user may alter the conditions of all the adjacent spaces, in turn transforming all other internal relationships.

Materiality- Hard and smooth/ Rigid and fluid: The harshness and almost unwelcoming attitude of the elevations, executed in fare face concrete, initially alienate visitors. It creates a hard shell that confines and protects the family activities. By its porosity, controlled by the users through sliding panels it allows the link with the external environment upon the users' will. As someone enters the house, however, he is taken by surprise: The softness and conviviality of the internal conditions generated by the central courtyard working in harmony with the network of peripheral courtyards make for a warm, nest-like atmosphere, flexible and easy to handle it for its inhabitants.

\section{COURTYARDS DEFINED AND SCULPTED BY LANDSCAPE AND NATURE}

Two houses on the outskirts of the small rural town of Dali were the starting point for an investigation into how the surrounding landscape redefines the main architectural element of the courtyard.

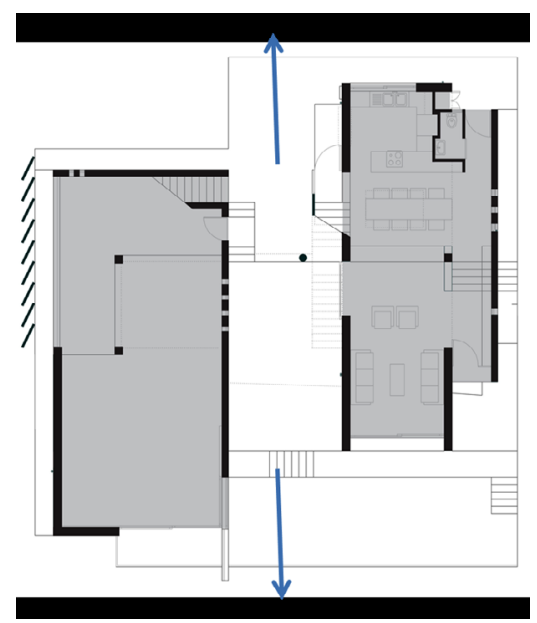

Figure 6: the landscape penetrates and defines the courtyard

Nature sculpts the sculptor's courtyard. The penetrating landscape (fig. 6)

The first case study combines a house with a sculptor's studio. The courtyard created here is flanked by the two elongated parts of the house and studio. The two narrow sides face the adjacent ascending hill, which descends through and sculpts the courtyard, inevitably focusing the attention on the faraway landscape and the prevailing mountain range of Pentadaktylos. The resulting levels of the courtyard correspond to the various domestic and artistic activities, which are enhanced by a network of movements that consequently transform the conditions of the courtyard.
The transformation of the 'atmospheres' of the courtyard culminates in the changes of the macro and micro natural environments, the vegetation, and the weather conditions, with the seasons taking on a main role in defining this specific courtyard. The spatial compositional elements of the courtyard respond to the climatic conditions, succeeding in directing and enhancing the air flow for cooling reasons, while protecting and shading from the summer sun, with the hovering volume of the bedrooms covering the central part of the courtyard.

The resulting transformations of the courtyard penetrate and enrich the internal conditions of the house and the studio through direct openings and loop systems of movements that are interwoven through it.

\section{Multishaped courtyard merged in the nature (fig. 7)}

This house was designed for a family with four children that wanted to live in close proximity to nature in a plot. The bedrooms and private spaces are organised as clusters of independent volumes that surround and envelop the family's communal activities.

A network of peripheral courtyards is formed between the solid private spaces, while its core is a structure gradually dissolves as its glazed transparent envelope opens up to its surroundings. It finally links and unifies the peripheral courtyard network, transforming it into a unified multi-shaped courtyard. It further extends into the surrounding natural environment, which in turn penetrates the courtyard.

In this case too, the changes in the natural surroundings - the orchard, the garden, the river bed, the eucalyptus cluster and the far away landscape - actively participate in and penetrate into the internal conditions and atmospheres of the courtyard and the living spaces of the house.

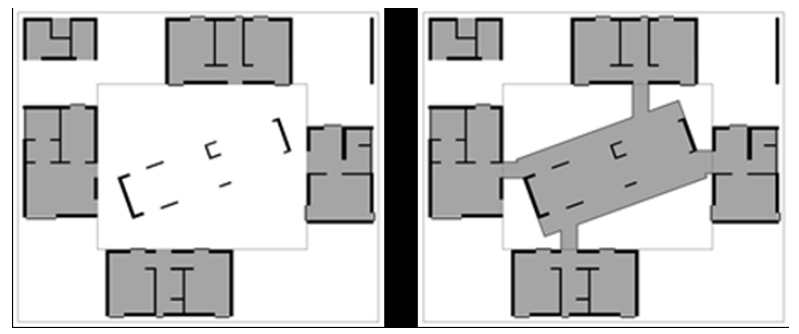

Figure 7: multishaped courtyards

\section{IMPLEMENTATION OF THE COURTYARD TYPOLOGIES IN URBAN SCALE ENVIRONMENTS}

The experimentation and research done for these realised case studies is rich material for pursuing this almost endless voyage of quest into the local architectural identity, focusing on the significance of the courtyard as the main spatial compositional element. The exploration continues on different scales and nature of use. The understanding of the courtyard spatial organisation can be seen as an urban feature pattern in horizontal and vertical development, continuously transformed in order to adjust to the natural, cultural, and social environment.

Decimal numbering of all sections is recommended (with the exception of the sections "ACKNOWLEDGEMENTS" and "REFERENCES"). If bold printing is not available to you, use underlining, instead, but only for subheadings and subsubheadings, not for Major Headings. 


\section{Courtyard qualities in a vertical development:}

An apartment complex in a new urban area of the seaside town of Pafos is developed vertically. The expected, conventional type of compact, one-floor apartments is translated into threestory houses with outdoor spaces as large as the indoor ones, while the lack of greenery due to the restricted size of the plot prompts the creation of a vertical park. This strategy could be achieved through the introduction of a new network of courtyards on all the floors of the building. Each courtyard is unique, defined by the relationship to the houses themselves, the vertical park and the faraway landscape. It is constantly transformed through the changes in vegetation and natural phenomena.

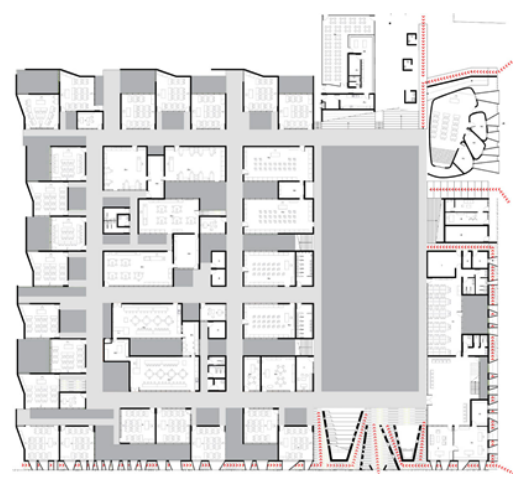

Figure 8: network of courtyards of different scales

\section{School environment with a dense network of courtyards of various scales (fig. 8)}

Inspired by the surrounding urban environment, which in itself may be viewed as a network of courtyards formed by the plot and house planning, a network of a wide variety of courtyards becomes the strong spatial composition and organisation of the school. The project was a competition entry that attempted to tackle innovatively the issue of a local identity for a school environment. The dense network of courtyards offers various courtyards in a wide range of scale, which are actively transformed by the presence of students and their various activities, in small or big groups.

\section{CONCLUSIONS}

In each case, new parameters filter the decisions that lead to a new architectural language for investigating and developing the architectural element of the courtyards, picking up the thread of history's wisdom.

Following this methodology, the final design cannot be predicted. It rather results from the meticulous analysis and clear understanding of the existing conditions [(Tarkofsky, 1986):in relationship to the response to the current and future needs of the people, town and the society. The inherent wise merits of the spatial organisations of the courtyard system ensure the respond to the cultural, social and environmental qualities of each project. In other words it carries and offers the richness and wisdom of the tradition. At the same time, the integration of gained knowledge and influence form the advanced technology and the methodologies of the spatial organisations developed in other countries may contribute the most for the improvement of the living conditions. The confluence of both parameters could be the generator of achieving innovative solutions for each specific situation. This approach becomes the way out to maintain, develop or (re)create an identity that derives from the tradition and targets to innovative solutions in order to respond to the constant changes and needs.

\subsection{References and/or Selected Bibliography}

Papacharalambous, G., 2001. The Cyprus House. Center of Scientific Researches, Nicosia

Pfeifer, G., Brauneck, P., 2008. Courtyard Houses, A Housing Typology. Birkhauser, Germany ()

Sinos, S., 1986. Types of Rural Dwellings in Cyprus. Acts of International Archaelogical Symposium. A, 1st, pp. 520--33

Lee, S. H., 1991. Continuity and consistency of the traditional courtyard house plan in modern Korean dwellings. Journal of the International Association for the Study of Traditional Environments Traditional dwellings \& settlements review. 3, 1, pp. 6-76

Khattab, O., Al-Saqabi, T., 2001. Revival of the traditional courtyard house model in contemporary housing in Kuwait. International journal for housing science \& its applications. 25, $4,301 \quad 10$

Danilo, D., 1997. The Walled City of Nicosia, Typology Study. UNDP United Nations Development Programme, Nicosia

Kyrris, C. P., 1996. History of Cyprus. Lampousa Publications, Nicosia

Schaar, K. W., Given, M., Theocharous, G., Bank of Cyprus, 1995. Under the Clock: Colonial Architecture and History in Cyprus, 1878-1960. Bank of Cyprus

Leventis, P.: Twelve Times in Nicosia: Nicosia, Cyprus, 11921570, 2005. Topography, Architecture, and Urban Experience in a Diversified Capital City. Cyprus Research Centre, Nicosia

Li, Y., Yan, X., 2010. Analysis on the Characteristics of Guanzhong Traditional Residential Courtyard --- Take Tang Courtyard in Xunyi County as an Example. Asian Social Science. 6, 3, 726

Bagneid, A. A, 1992. The microclimate of courtyards: Experiments on three evaporative cooling floor treatments. Ekistics. 59, 354 355, 217-29

Serghides, D., 2010. The Wisdom of Mediterranean Traditional Architecture Versus Contemporary Architecture - The Energy Challenge. The Open Construction and Building Technology Journal. 4, 2010, pp. 2938

Tarkofsky, A., 1986. Sculpting in Time. Nefeli Greek publication, Athens 
